\title{
The'microanatomy of the alveolar duct system
}

\author{
W. F. W H I M S T E R ${ }^{1}$ \\ Department of Pathology, University of the West Indies, Kingston, Jamaica
}

\begin{abstract}
Observations made on wet and dry slices of inflated human lungs taken at necropsy have been used to give a more complete account of the alveolar duct system, the basic respiratory structure. This includes consideration of the central spiral alveolar duct fibre, which is thought to consist of elastic and collagen, the spiral arrangement of alveoli and the special nature of the terminal alveolus, the interdigitation of alveoli from adjacent alveolar ducts and the arrangement of pulmonary arterioles. This suggests that the alveolar duct system is specifically designed so that changes in volume are not accompanied by changes in alveolar surface area.
\end{abstract}

Of the small holes seen in the cut surface of the fixed inflated lung (Fig. 1) the vast majority are found to be alveolar ducts, separated by translucent brownish alveolar tissue. The structure of the alveolar ducts, which, with the alveoli opening off them, make up the basic respiratory unit of the lung, has been considered by many authors. The diagrams of Miller (1947) represent an older opinion, and those of Oderr (1964) a more recent view. Further observations are reported here from which a more complete account of the alveolar duct system and its relationship to pulmonary vessels and to other alveolar duct systems can be inferred.

\section{METHODS}

Normal and diseased lungs were obtained from unselected adult patients of various ages and of Negro extraction coming to necropsy at the University Hospital of the West Indies. Some of the lungs were inflated with $10 \%$ formalin via the main bronchus, sliced after 24 hours into four or five sagittal slices, $1.5 \mathrm{~cm}$. thick, and examined with a dissecting microscope, and blocks were taken for histology. Rapid paper sections (Whimster, 1969) were then made. Other lungs were fixed and dried in inflation by the method of Blumenthal and Boren (1959) with or without prior injection of the arteries and veins with a barium-gelatine solution. The dried lungs were sliced on a bacon slicer at approximately $750 \mu$, and examined unstained between transparent plastic sheets perforated at $1-\mathrm{cm}$. intervals under an ordinary binocular microscope; photomicrographs were taken as required. Adult lungs yielded up to 100 slices.

1 Present address : Department of Pathology, Royal Free Hospital, London, W.C.1.
Selected areas of the dried slices were cut out, soaked in water in a vacuum chamber to remove bubbles, and stained. They were mounted on $3 \times 1$ in. slides by covering the $750 \mu$ thickness with Maraglas (Polysciences Inc., Rydal, Pennsylvania 19046, U.S.A.), removing bubbles in a vacuum chamber for 1 to 2 hours, putting on a coverslip and setting in an oven at $52^{\circ} \mathrm{C}$. overnight.

For these anatomical studies, normal lungs were selected as far as possible: they were normal to inspection and palpation and weighed less than the mean for normals of that size and sex (such means and standard deviations had been obtained from an analysis of 340 lungs out of 2,038 from our necropsy files in which no abnormality was found grossly, on slicing, or on histology).

\section{OBSERVATIONS}

The smallest holes in the wet lung slice or paper section (Fig. 1) were readily seen in the dry lung slice (Fig. 2). The alveoli opening off all sides of the holes confirmed that the latter were alveolar ducts. Bronchi and bronchioles were easily distinguished from ducts which had alveoli arising on all sides. Vessels could be identified either by blood remaining in them or by the injection mass. The spiral nature of the alveolar duct (Oderr, 1964) was best appreciated by focusing up and down through the $750 \mu$ thickness on ducts which were cut transversely or slightly obliquely (Fig. 2). This three-dimensional impression was more striking under the microscope than can be seen in the black-and-white photograph. The contour of the spiral was determined by the thick spiral fibre between the turns of which the alveoli opened. This is shown diagrammatically in Fig. 3, and can be seen arrowed in Figure 2. When stained by the 


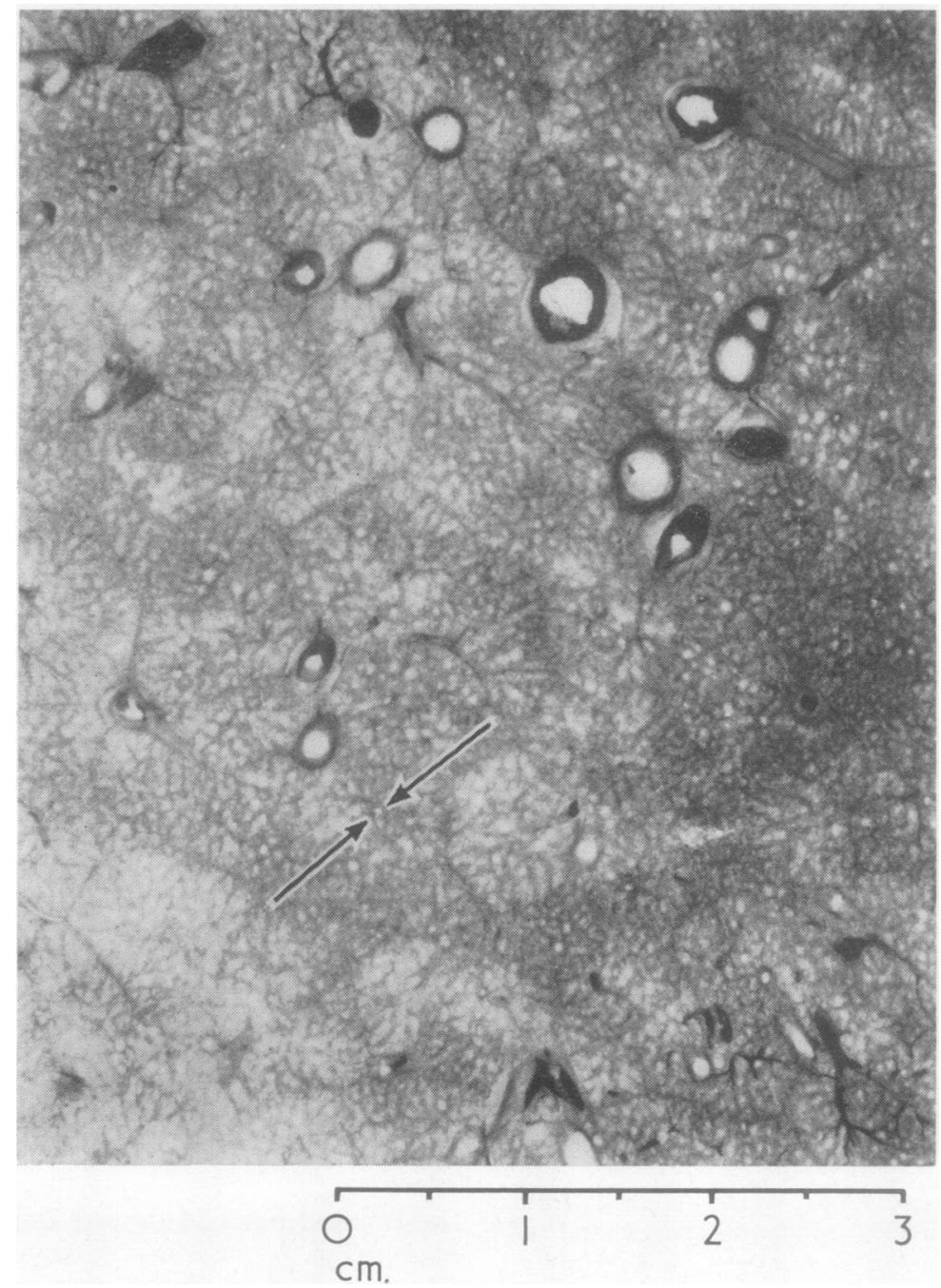

FIG. 1. Formalin-inflated normal lung showing parenchyma with numerous alveolar ducts (arrowed) in contrast with larger vessels and bronchi (Rapid paper section $\times 2$ ).

Verhoeff-van Gieson technique (Culling, 1963 (a)), the central spiral fibre had a streaky red and black appearance, supporting the view (Pierce, 1968) that it was composed of a mixture of elastic and collagen (Fig. 4). The lines where alveolar walls adjoined also gave the same staining reaction. No other collagen could be demonstrated in normal areas, although fine reticulin fibres could be seen (Fig. 5) using the Gordon and Sweet's silver staining technique (Culling, 1963 (b)). The reticulin fibres did not show the association with capillaries suggested by Spencer (1968). Fine elastic fibres could be seen fanning across the alveolar walls among the capillaries (Fig. 6).

The alveolar network of capillaries could be clearly seen (Fig. 6), and occasionally it was possible to see small vessels dividing up into capillaries. The small vessels ran between the alveolar duct system (Figs. 3 and 7), thus reaching the backs of the alveoli. Both arterioles and venules could be identified.

The cell and nuclear detail was well preserved 


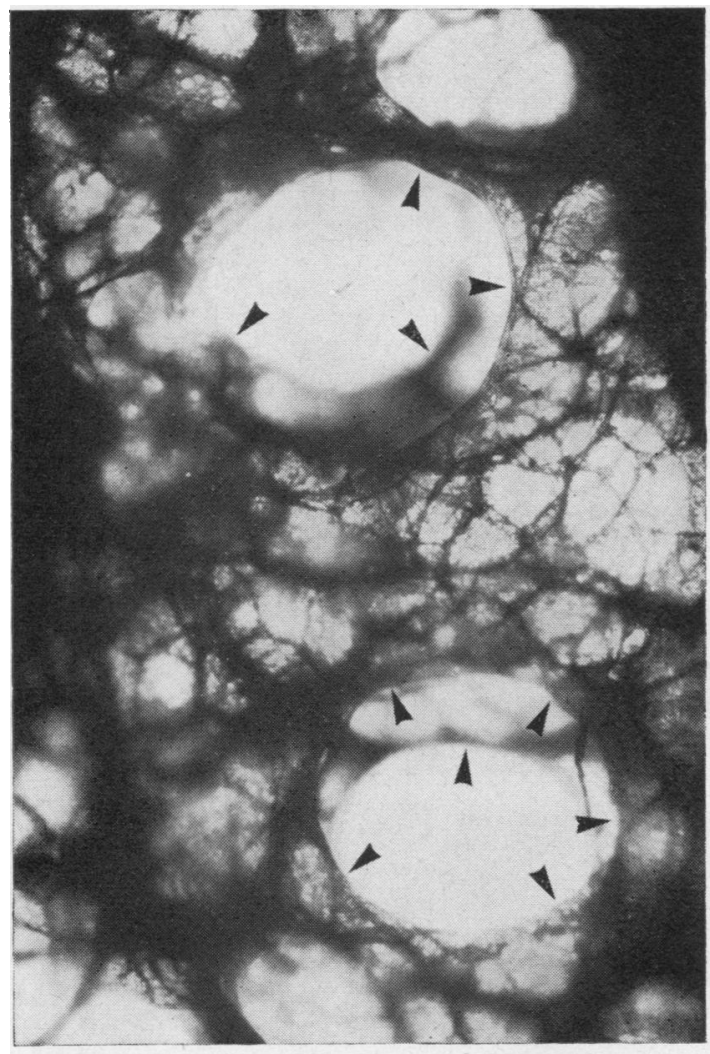

FIG. 2. A slice of inflated, fixed, dried lung showing two adjacent spiral alveolar ducts (spiral fibre arrowed) and alveoli (unstained $\times 52$ ).

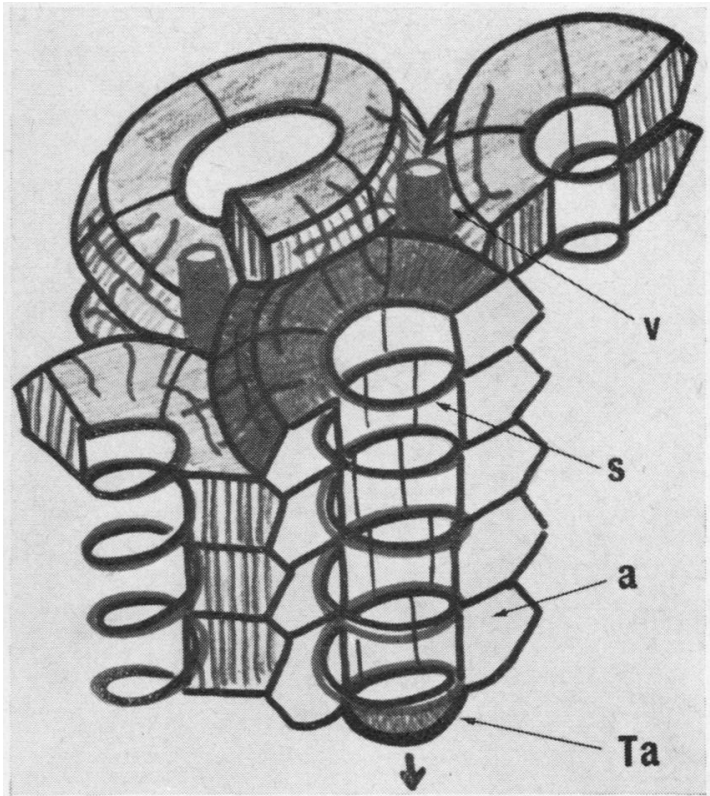

FIG. 3. A diagram showing parts of four interdigitating alveolar duct systems, including spiral collagen-elastic fibres ( $s$ ), alveoli (a), a terminal alveolus (Ta) and vessels (v).

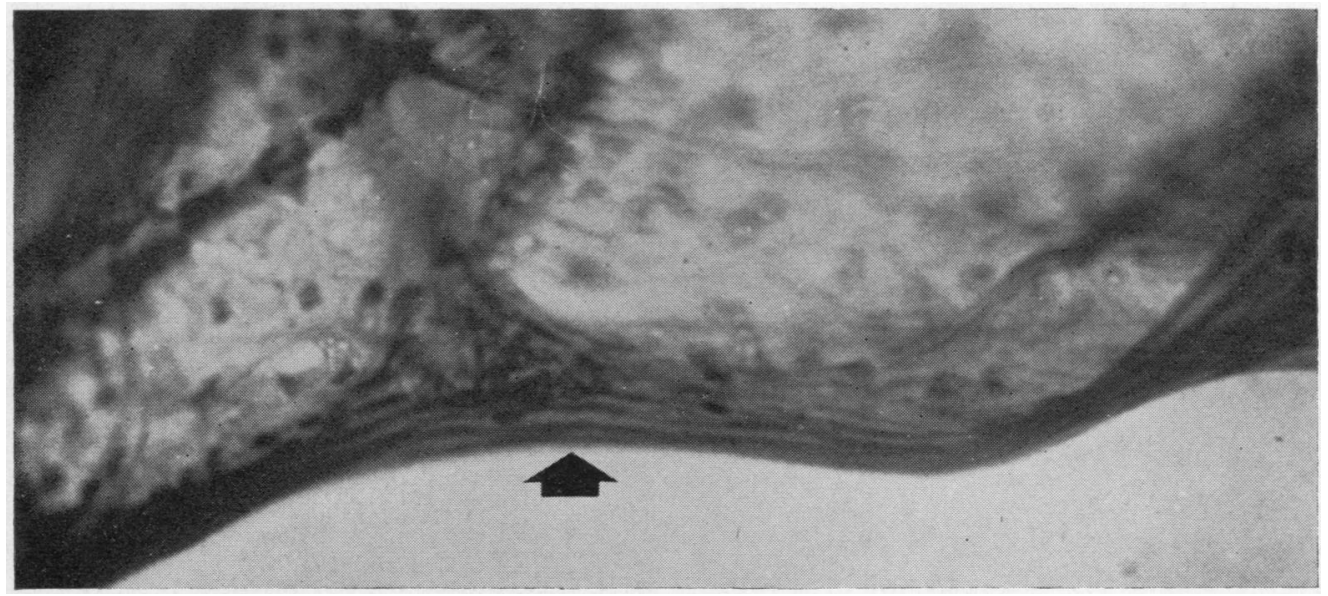

FIG. 4. An alveolar duct fibre (arrowed) composed of elastic and collagen (dried lung, Verhoeff-van Gieson $\times 500$ ). 


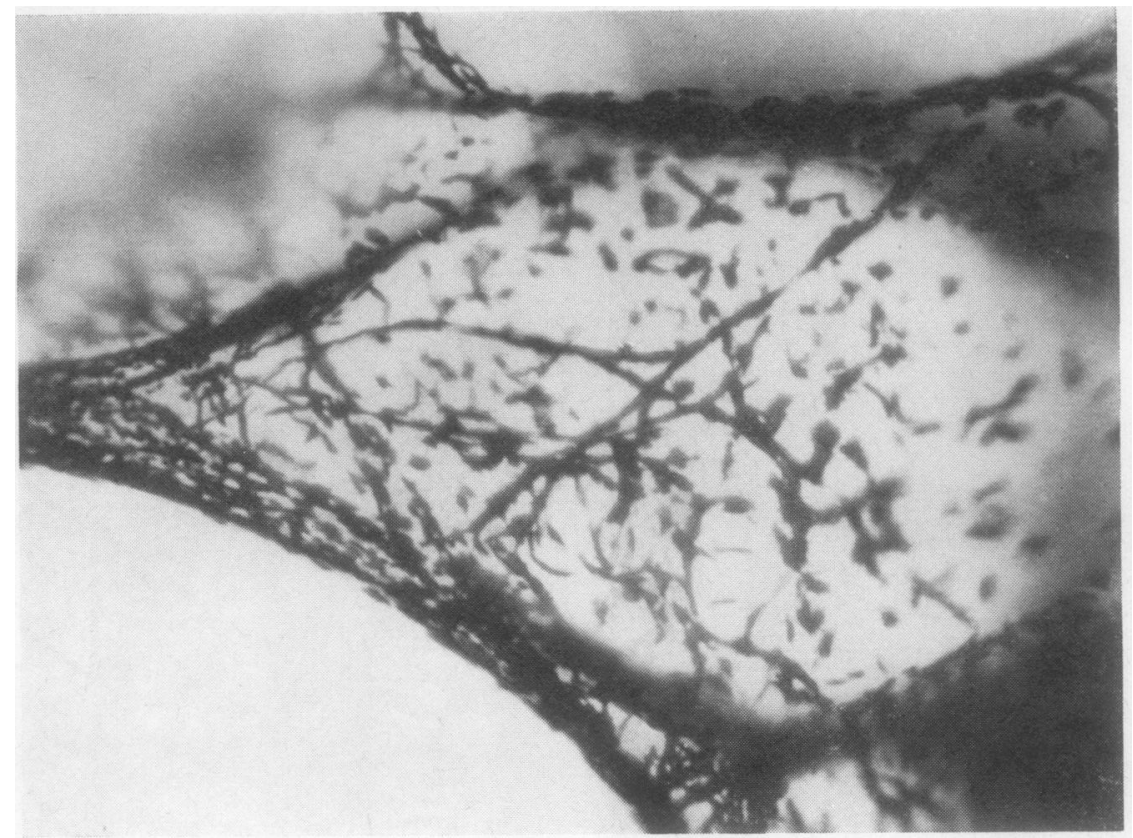

FIG. 5

FIG. 5. An alveolar wall showing reticulin fibres (dried lung, reticulin stain $\times 500$ ).

FIG. 6. Alveolar wall showing elastic-collagen boundaries, fine elastic fibres and capillary network (dried lung, Verhoeff-van Gieson × 730).

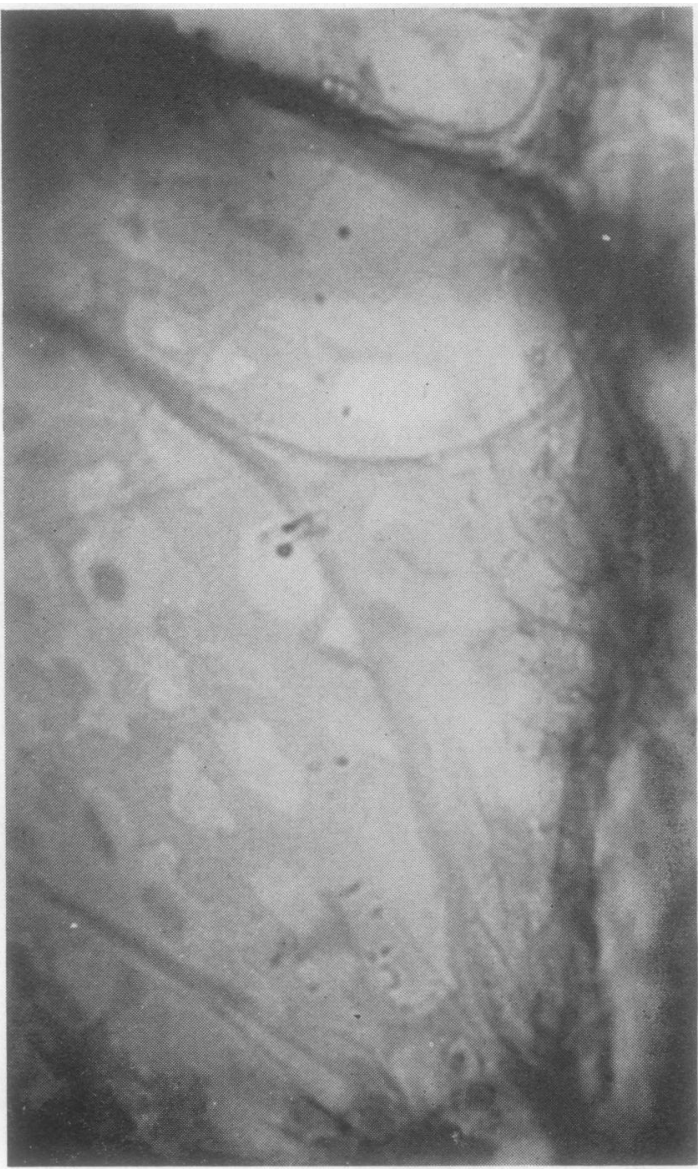




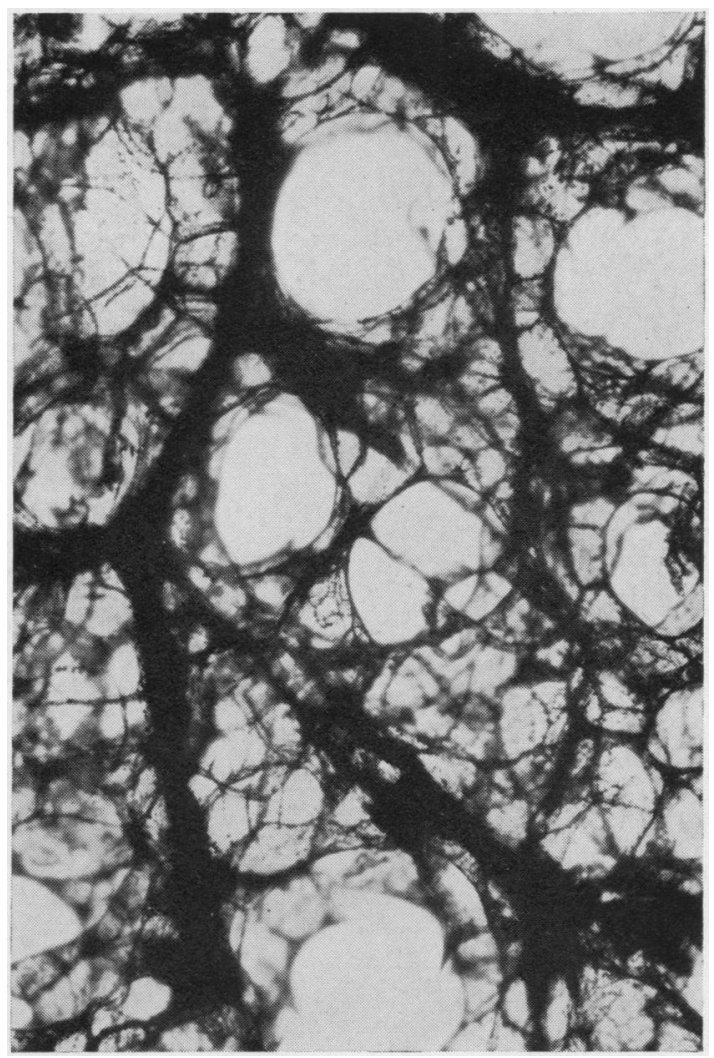

FIG. 7. Small vessels passing between alveolar duct systems (dried lung, reticulin stain, $\times 52$ ).

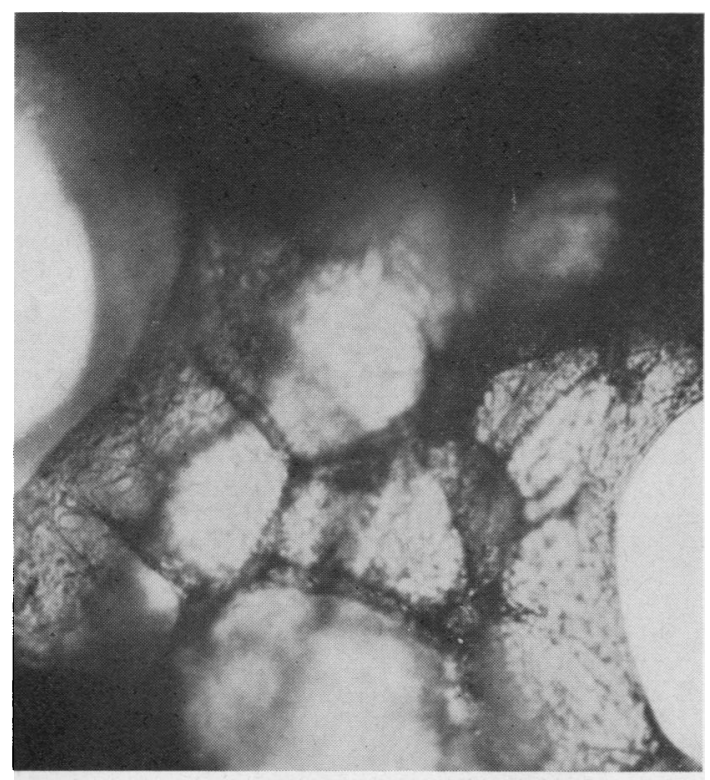

FIG. 8. Interdigitation of alveoli from adjacent alveolar duct systems (dried lung, unstained, $\times 130$ ).

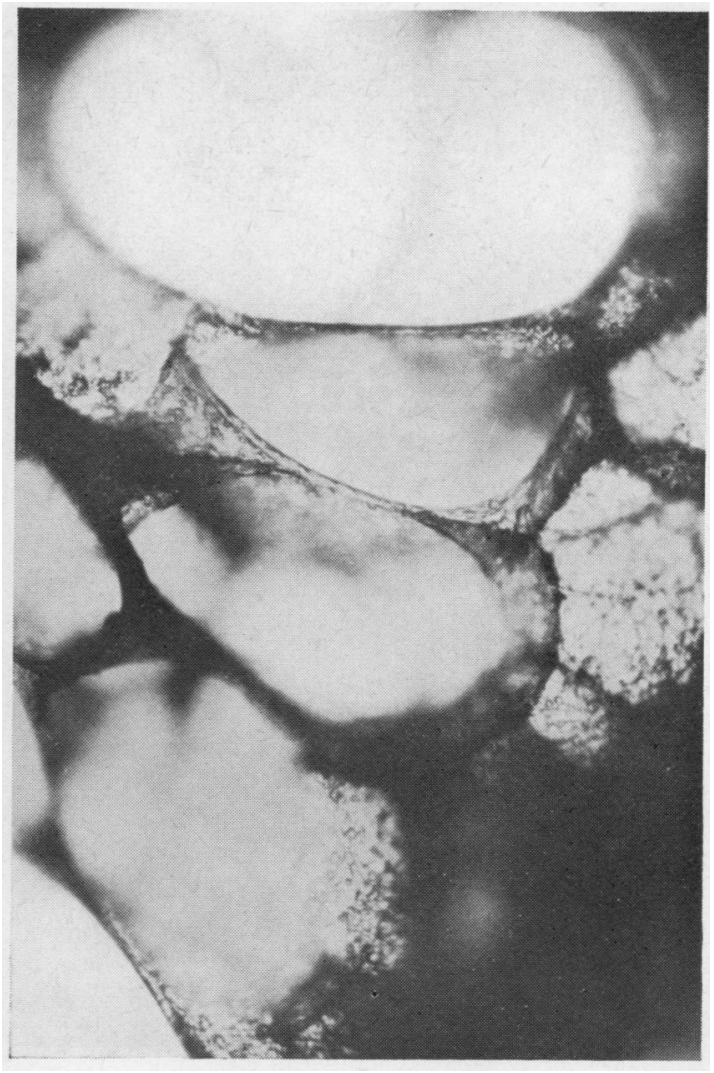

FIG. 9. Elliptical alveoli (dried lung, unstained, $\times 130$ ).

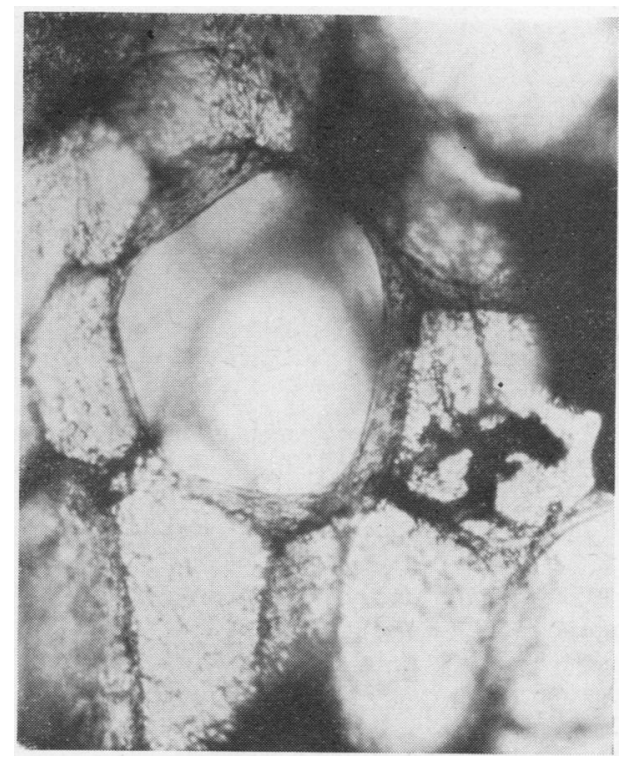

FIG. 10. A spherical alveolus (dried lung, unstained, $\times 130)$. 


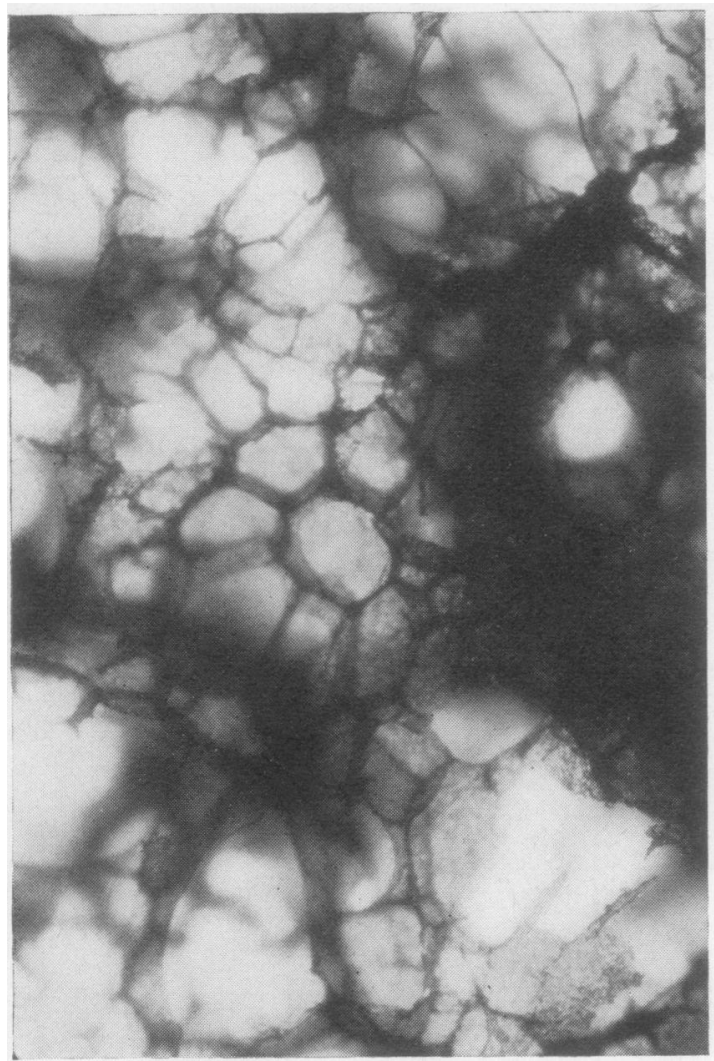

FIG. 11

FIG. 11. The end of an alveolar duct showing the terminal alveolus, seven alveoli in the last turn of the spiral and interdigitation with neighbouring systems (dried lung, unstained, $\times 52)$.

FIG. 12. A complete alveolar duct in histological section (haematoxylin and eosin, $\times 130$ ).

FIG. 13. Alveolar ducts in pneumonia (dried lung, unstained, $\times 52$ ).

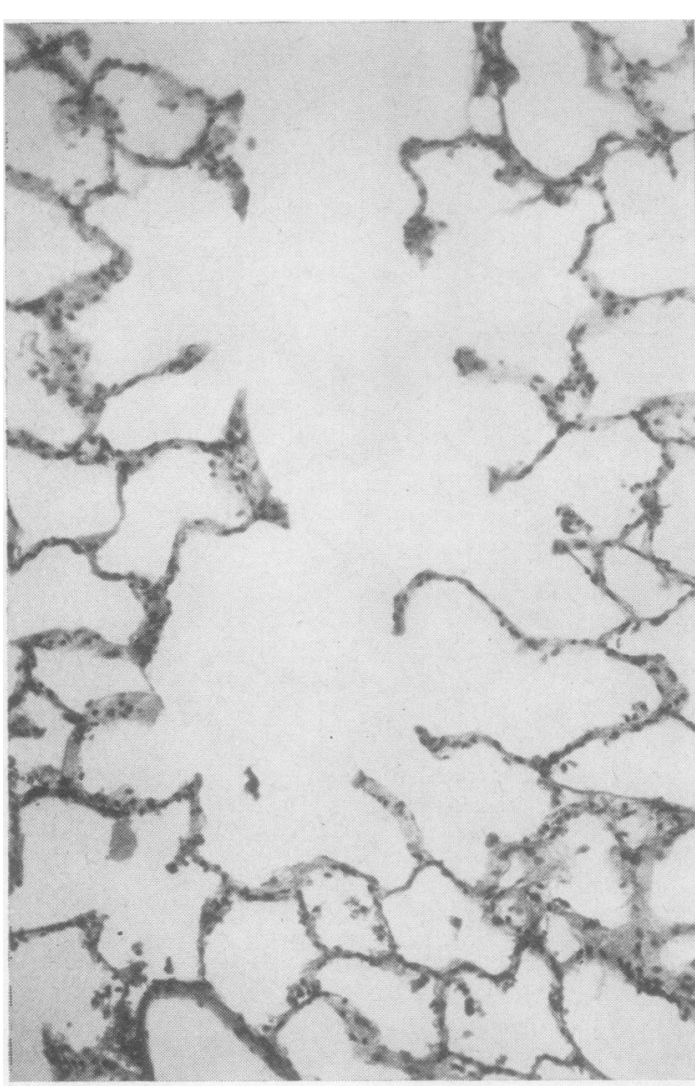

FIG. 12

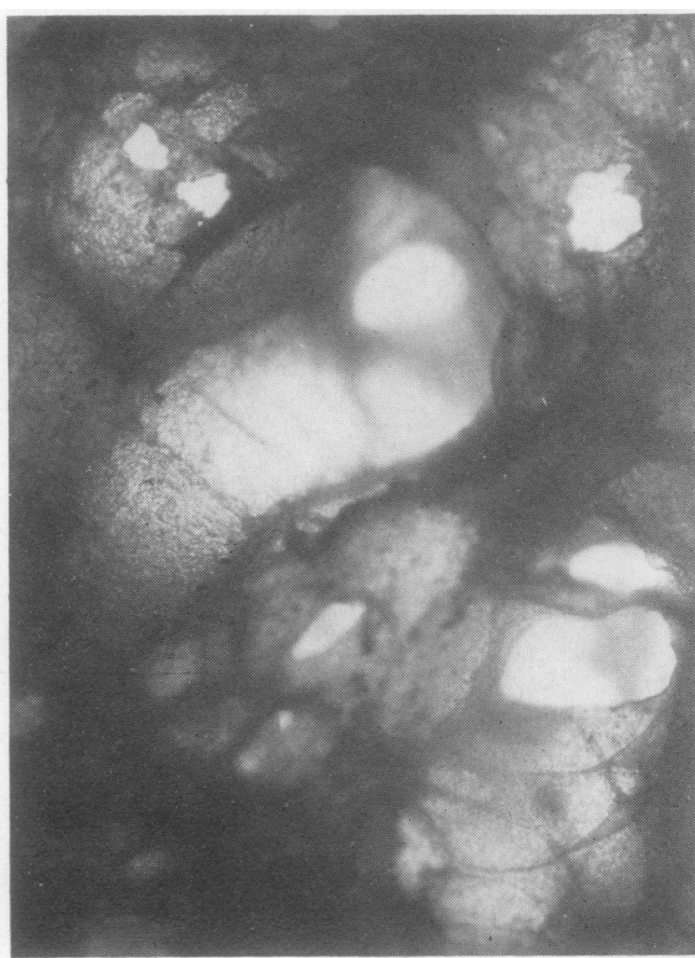

FIG. 13 
in haematoxylin and eosin stained pieces of dried lung, so that eosinophilic granular pneumocytes could be seen scattered at random through the alveolar walls, not particularly at the corners of adjoining alveolar walls, among the non-granular alveolar cells. Alveolar macrophages containing brown granules could be recognized.

There appeared to be a great variation in the size and shape of the alveoli. Measurements in the three-dimensional material in varying degrees of inflation were not regarded as accurate but are given here as a rough guide. In one adult lung (female, 70 years) alveolar openings ranged from $180 \mu$ by $240 \mu$ for the smallest to $340 \mu$ by $360 \mu$ for the largest, with a mean of $240 \mu$ by $310 \mu$. The alveolar ducts, which appeared larger when seen in transverse section, gave a mean diameter of $470 \mu$ (range $270-600 \mu$ ). It was clear that, where alveolar ducts were seen together in the right plane, the alveoli arising from one interdigitated with those of others (Figs 8 and 11), as shown diagrammatically in Figure 3.

The shape depicted in Fig. 3 for the majority of alveoli is similar to that chosen by. Weibel (1963) for mathematical calculations, but his observation that 'no well-defined geometric form has been found to represent the "typical alveolus" appeared to be confirmed in the present study. For, although the shape depicted was often seen, so were many other variously inflated elliptical alveoli, and occasionally spherical alveoli, of which Figs 9 and 10 show examples.

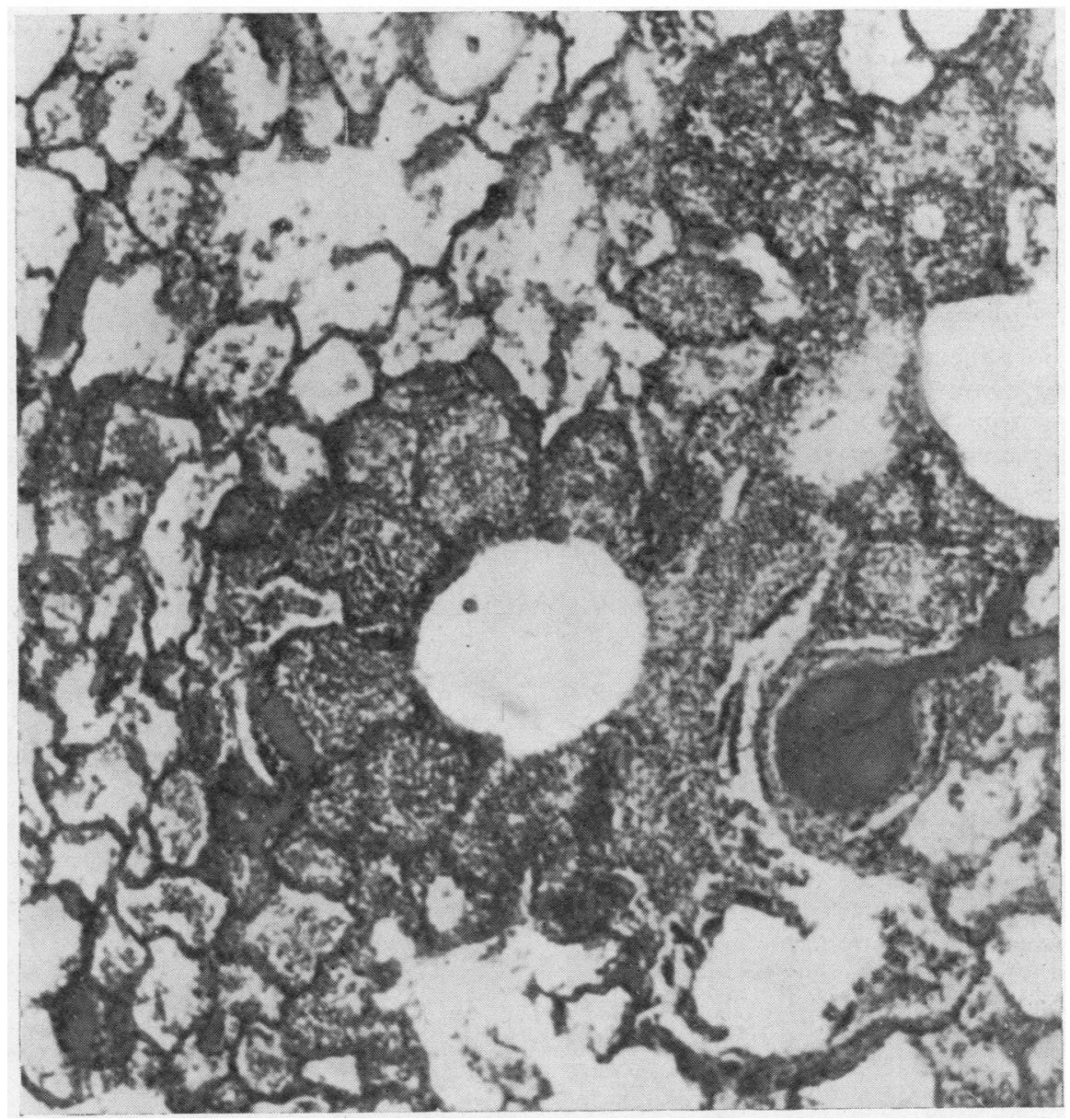

FIG. 14. An alveolar duct surrounded by six exudate-filled alveoli, in histological section (haematoxylin and eosin, $\times 80$ ). 
The spherical alveoli were at first thought merely to be over-inflated alveoli, but it became apparent that such alveoli could also be found at the ends of alveolar duct spirals (Figs 3 and 11). It has not yet been possible to isolate such alveoli for certain in stained preparations to see if there is an increase in alveolar wall fibres, as might be expected if the terminal alveolus has a mechanical rather than a respiratory function.

The alveolar openings usually had four corners and straight or convex edges, but en face the alveolar walls were often pentagonal, as Boren (1962) noted. Walls of six sides were also fairly frequent, and four- or seven-sided walls sometimes occurred but triangular walls were not seen.

The number of alveoli in a complete turn of the spiral was not easy to count as it was often difficult to see all of them, but between five and eight was the usual number (Figs 11 and 14). It was impossible to determine the number of turns of the spiral because the duct had to be cut before the observer could see into it (transverse cutting precluded counting and longitudinal cutting was extremely difficult to interpret). From histological preparations, of which Fig. 12 is an example, it would seem that the number of turns of the spiral is variable, perhaps four or five turns being normal.

In areas of pneumonic consolidation, which were not too dense to prevent light passing, the spiral arrangement of the alveoli was not so clear (Fig. 13), presumably because the inflammatory exudate tended to fill the alveoli initially while air could still pass up and down the alveolar duct, as is seen histologically in Figure 14.

\section{DISCUSSION}

These observations and interpretations are open to numerous criticisms. For example, despite careful selection, the lungs may have been abnormal in ways that never became apparent to the observer. The techniques described introduce many artefacts. Individual alveolar duct systems were bound to have been inflated to different degrees, for no external control of the inflating pressure, whether constant or intermittent, could control the inflation of individual alveolar ducts which were probably unevenly inflated in life. Fixation itself produces distortion and shrinkage. Slicing introduces cutting artefacts. Sampling errors were reduced as far as possible by systematically examining the dry lung slices at $1-\mathrm{cm}$. intervals.

The recent development of ideas about the microanatomy of the lung (Blumenthal and Boren,
1959 ; Boren, 1962 ; Weibel, 1963 ; Oderr, 1964 ; Pierce, 1968) has come from the use of new techniques in the examination of inflated excised lungs from which the living state can be approximately deduced. The present study attempts to carry this development a stage further. It offers an improved model of the alveolar duct system which also takes into account the relationships of alveolar duct systems to each other and to the pulmonary blood vessels.

With regard to the central alveolar duct fibre, Orsos (1907) described an inter-weaving of collagenous and elastic fibres, and this mixture has been confirmed under the electron microscope

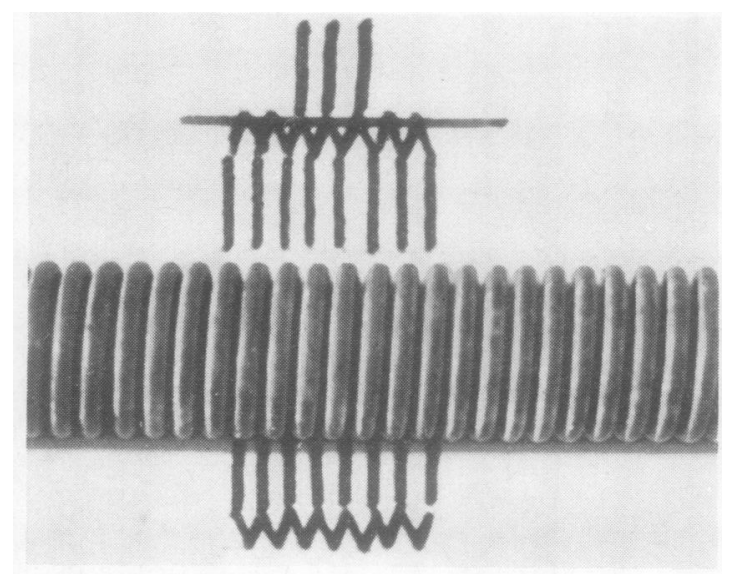

(a)

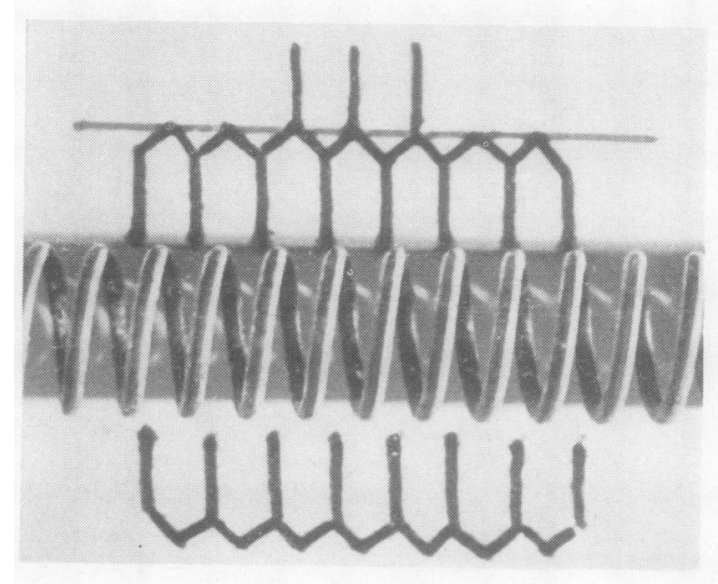

(b)

FIG. 15. Diagrammatic representation of alveoli (a) folded down in expiration and $(b)$ unfolded in inspiration. 
(Pierce, 1968). Pierce also realized that this composition would give little extensibility but great tensile strength, so that a coil of it could function like 'an old-fashioned door spring'.

Oderr (1964) drew his alveoli opening off the central corridor formed by the spiral but drew them as 'parallelepipeds', box-like structures with one open side, which folded and unfolded, but without mention of what happened to the back. Very few, if any, alveoli in the present study have been rectangular, whereas pentagonal sides have been common. Observation suggests, therefore, that the alveolus more usually has the shape shown in Figure 3. This enables it to fold and unfold without stretching, in the manner shown in Figure $15 \mathrm{a}, \mathrm{b}$. The paucity of fibres in the alveolar wall supports the idea that the alveolar wall is not subjected to more than minimal stretching.

Weibel (1963) calculated that in an adult lung there were 300 million alveoli, 14 million alveolar ducts and a mean of 21 alveoli per alveolar duct. The present study suggests that there are between 20 and 40 alveoli per alveolar duct, with a higher mean of about 30 , so that borrowing the figure of 300 million alveoli (rather than the alveolar duct figure with which Weibel was less concerned), a total of 10 million alveolar ducts is obtained.

The working of the alveolar duct system, as now conceived, is that in inspiration the pull exerted evenly over the periphery of the lung by the pleural surface tension as the thoracic volume enlarges extends the alveolar duct spirals in a linear fashion rather than with the rotatory movement of a door spring. So the alveoli unfold, the most peripheral first, their surface tension reduced by surfactant, with minimal change in surface area. Gas exchange takes place between the inspired air and the capillary network.

In this respect the spherical alveoli seen at the ends of alveolar ducts and elsewhere are presumably unphysiological. If alveoli were like balloons, their walls would stretch during inspiration and the capillaries would be obliterated at the time of gas exchange. The alveolar duct system appears designed specifically to prevent this and to allow maximal changes in volume with minimal changes in the surface area. If sufficient alveoli become overinflated and behave like balloons, presumably overall ventilation-perfusion relationships would be affected and this may be the case in panacinar emphysema. It may, however, be permissible in the terminal alveoli of the alveolar ducts which nevertheless represent perhaps $3 \%$ (1 in 30$)$ of the alveoli.

The present model considers the relationship of one alveolar duct system with its neighbours by interdigitation of their alveoli so that the greatest number of alveoli can be contained in each lung lobule. Arterioles and venules pass along the backs of the alveoli between the alveolar duct systems, with access to the walls of several systems. Those vessels which continue from the bronchioles in the longitudinal axis of the alveolar ducts must have some tortuosity in expiration to allow them to straighten as the duct lengthens in inspiration.

The model suggested probably does not take into account all the variations in size and shape of the respiratory structures seen in these preparations, even when local differences in inflation are taken into account. It is quite possible that alveoli of different contour function differently; some alveolar duct systems may be designed to be held in reserve.

One is left with the impression that 10 or 14 million alveolar duct systems per lung allow plenty of scope for variation and present considerable problems to the investigator.

I am indebted to Professor G. Bras, University of the West Indies, for his continued encouragement and support; to Mr. L. Facey and Mrs. J. J. Audretsch for technical assistance; and to Mr. L. Dawson and Mr. T. Haughton for photography.

\section{REFERENCES}

Blumenthal, B. J., and Boren, H. G. (1959). Lung structure in three dimensions after inflation and fume fixation. Amer. Rev. resp. Dis., 79, 764.

Boren, H. G. (1962). Alveolar fenestrae. Amer. Rev. resp. Dis., 85,328 .

Culling, C. F. A. (1963). Handbook of Histopathological Techniques, 2nd ed., (a) p. 342; (b) p. 347. Butterworths, London.

Miller, W. S. (1947). The Lung, 2nd ed., Ch. 4. Thomas, Springfield, Illinois.

Oderr, C. (1964). Architecture of the lung parenchyma. Studies with a specially designed X-ray microscope. Amer. Rev. resp. Dis., $90,401$.

Orsós, F. (1907). Utber das elastische Gerüst der normalen und der emphysematossen Lunge. Beitr. path. Anat., 41, 95. Quoted by

Pierce, J. A. (1968). In The Lung, p. 41. Ed., Liebow, A. A., and Smith, D. E. Williams and Wilkins, Baltimore. Spencer, H. (1968). In The Lung, p. 134. Ed., Liebow, A. A.. and Smith,
D. E. Williams and Wilkins, Baltimore.

Weibel, E. R. (1963). Morphometry of the Human Lung. Springer-

Whimster, W. F. (1969). Rapid giant paper sections of lung. Thorax,
24,737. 